\title{
Ribozymes and Riboswitches: Modulation of RNA Function by Small Molecules ${ }^{\dagger}$
}

\author{
Jinwei Zhang ${ }^{1}$, Matthew W. Lau², and Adrian R. Ferré-D'Amaré1,2,* \\ ${ }^{1}$ Howard Hughes Medical Institute, Fred Hutchinson Cancer Research Center, 1100 Fairview \\ Avenue North, Seattle WA 98109-1024, USA \\ 2 Division of Basic Sciences, Fred Hutchinson Cancer Research Center, 1100 Fairview Avenue \\ North, Seattle WA 98109-1024, USA
}

\begin{abstract}
Diverse small molecules interact with catalytic RNAs (ribozymes) as substrates and cofactors, and their intracellular concentrations are sensed by gene-regulatory mRNA domains (riboswitches) that modulate transcription, splicing, translation, or RNA stability. Although recognition mechanisms vary from RNA to RNA, structural analyses reveal recurring strategies that arise from the intrinsic properties of RNA such as base pairing and stacking with conjugated heterocycles, and cation-dependent recognition of anionic functional groups. These studies also suggest that, to a first approximation, the magnitude of ligand-induced reorganization of an RNA is inversely proportional to the complexity of the riboswitch or ribozyme. How these small molecule bindinginduced changes in RNA lead to alteration in gene expression is less well understood. While different riboswitches have been proposed to be under either kinetic or thermodynamic control, the biochemical and structural mechanisms that give rise to regulatory consequences downstream of small molecule recognition by RNAs mostly remain to be elucidated.
\end{abstract}

Ribozymes and riboswitches starkly demonstrate the ability of RNA to fold into complex structures that position functional groups with exquisite precision. The former are catalytic RNAs; the latter, cis-acting regulatory mRNA domains that respond to the intracellular concentration of small molecule metabolites and second messengers [the first example of a trans-acting riboswitch RNA was recently described (1)]. In vitro, both ribozymes and riboswitches can function in the absence of protein cofactors, although some catalytic RNAs are known to require chaperones [reviewed in (2)] for in vivo activity, and riboswitches ultimately need to interface with the rest of the gene expression (transcription, splicing, translation, or RNA degradation) machinery for their small molecule-dependent regulatory activity to become manifest. Over the past decade, structural analyses have shed light on the mechanism of small molecule recognition by ribozymes (as substrates and coenzymes) and riboswitches (as regulatory signals). We review the state of knowledge of small molecule recognition by RNA, and how small molecule binding gives rise to genetic regulation.

\section{Ligand recognition by natural and artificial aptamers Structures of riboswitch aptamer domains}

At the time of writing, structures of the ligand-binding [or "aptamer" (3)] domains of representatives of eleven different classes of naturally-occurring riboswitches have been

\footnotetext{
$\dagger^{\dagger}$ This work is supported by the Howard Hughes Medical Institute (H.H.M.I.), an NIH grant (GM63576) to A.R.F., and a Croucher Foundation Fellowship to M.W.L. A.R.F. is an Investigator of the H.H.M.I.

*To whom correspondence should be addressed, aferre @fhcrc.org; telephone (206) 667-3621; facsimile (206) 667-3331.
} 
reported: cyclic diguanylate (c-di-GMP) (4,5), flavin mononucleotide (FMN) (6), $\mathrm{glmS}$ $(7,8)$, lysine $(9,10)$, preQ 1 (11-13), magnesium ion (14), purine (guanine and adenine) $(15,16)$, S-adenosylmethionine Class I (SAM-I) (17), SAM-II (18), SAM-III (19), and thiamine pyrophosphate (TPP) (20-22). The aptamer domains of these riboswitches all adopt distinctly different structures. There are no discernible evolutionary relationships between different classes of riboswitches, making it uncertain whether each class descends from an independent evolutionary ancestor, or if sequence and structure divergence have proceeded too far for lineage relationships to be apparent.

Riboswitch aptamer domains can be classified on the basis of structural features. For instance, and analogous to ribozyme classification $(23,24)$, riboswitch aptamers can be divided into those that are organized around multi-helical junctions, and those whose architectures result from pseudoknot (25) formation. The c-di-GMP, purine, SAM-III and TPP aptamer domains are all 3-helix junctions, and except for SAM-III, form Y-shaped structures (26) stabilized by distal interactions between two of the helices (Figure 1A). The $\mathrm{Mg}^{2+}$ riboswitch contains two 3-helix junctions. The lysine riboswitch structure is organized around a 5-helix junction, and that of the FMN riboswitch, around a 6-helix junction. The latter is notable for a series of loop-loop interactions that result in a pseudo-symmetric molecule that recognizes its ligand asymmetrically. The SAM-II and preQ $_{1}$ riboswitch aptamer domains are simple H-type pseudoknots (Figure 1B). The SAM-I riboswitch aptamer domain is organized around a 4-way helical junction, but its folded structure also contains a pseudoknot (Figure 1C). Finally, the $\mathrm{g} \operatorname{lmS}$ riboswitch-ribozyme is comprised of three pseudoknots. Its minimal core, sufficient in vitro for ligand recognition and catalysis, comprises a nested double pseudoknot. A peripheral domain folds as an H-type pseudoknot that itself functions as a 3-helix junction (Figure 1D).

\section{Ligand recognition strategies of riboswitches}

Given the diversity of riboswitch aptamer domain architectures, it is not surprising that the specific mechanisms employed by these RNAs to recognize their cognate ligands vary greatly. Nonetheless, the characteristic chemical properties of RNA give rise to several recurrent molecular recognition strategies. Recognition of TPP by its cognate riboswitch illustrates three of these: base stacking, base pairing, and metal ion-mediated binding (Figure 2). TPP is bound in an extended conformation and spans two of the three helices of the aptamer domain. The pyrimidine ring of TPP is recognized through stacking and formation of a base pair-like interaction (27) with the sugar edge of G40. The central thiazole ring of TPP makes only van der Waals interactions with the RNA. The pyrophosphate of TPP is essential for recognition by the riboswitch. The RNA does not recognize the pyrophosphate directly; rather, it binds it as a chelate with two partially hydrated divalent cations. Indeed, most of the interactions between RNA and pyrophosphate atoms are mediated by the two divalent cations or by their hydration sphere $(20,21)$ (Figure 2). Crystal structures of the riboswitch bound to thiamine monophosphate (TMP), against which it discriminates at least 30-fold (28), revealed that the riboswitch binds to the shorter ligand using essentially the same interactions. This is made possible by a slight compaction of the riboswitch, which brings the two helices that recognize the opposite ends of the ligand closer, and by coordination of the single phosphate of TMP with two divalent cations that in turn make interactions with the RNA similar to those made by the two cations chelated by TPP (20).

Stacking and base pairing are predictably common in riboswitches that recognize nucleobases or other planar heterocycles (27). The purine, c-di-GMP and $\mathrm{preQ}_{1}$ riboswitches all recognize guanine or guanine-containing ligands using these strategies, yet the specific nucleobase interactions employed are different in each case $(4,5,11,16)$ (Figure 3 ). In all cases, stacking of the ligand between nucleobases is important, but it has been noted that the 
stacking interactions employed by the purine riboswitch are sub-optimal. This may have the consequence of increasing the contribution to specificity of base pairing (29). Indeed, substitution of C74 (Figure 3A) to $\mathrm{U}$ is sufficient to switch the ligand specificity of the riboswitch from $\mathrm{G}$ to $\mathrm{A}$ (30). Consistent with their architectural diversity, the three structurally characterized classes of SAM riboswitches employ different base-pairing schemes to recognize the adenine moiety of their ligand. Their strategies for binding the other elements of SAM are also distinctly different $(18,19)$. The FMN riboswitch recognizes the edge of the isoalloxazine ring of FMN that resembles a uracil through formation of a base pair-like interaction with an adenosine residue of the RNA (6).

Reminiscent of the TPP riboswitch, the FMN riboswitch employs a tightly bound metal ion to recognize the phosphate moiety of FMN. Like the TPP riboswitch, the FMN riboswitch can employ a variety of divalent cations in vitro to mediate recognition of the phosphate of its ligand (6). The phosphate groups of c-di-GMP and glucosamine-6-phosphate (GlcN6P) are also recognized by their respective cognate riboswitch as divalent metal ion chelates $(5,7)$. Metal ion-mediated ligand recognition by riboswitches is not limited to phosphatecontaining ligands. The lysine riboswitch has a strict dependence on $\mathrm{K}^{+}$for ligand recognition. The structure shows that $\mathrm{K}^{+}$coordinates the carboxylate of the bound lysine, and the cation in turn makes inner-sphere coordination with RNA functional groups (9). Lysine binding is 50-100 fold weaker if $\mathrm{K}^{+}$is replaced with $\mathrm{Na}^{+}$or $\mathrm{Mg}^{2+}(9)$. The $\mathrm{preQ}_{1}$ riboswitch discriminates $\sim 25$-fold against guanine, from which its cognate ligand differs by the exocyclic aminomethyl group (and the deaza substitution at position 7) (Figures 3A, 3B) (31). The cocrystal structure shows that the exocyclic amine participates in tetrahedral hydrogen bonding with three ligands: a non-bridging phosphate oxygen, the carbonyl oxygen of G5, and a hydration water of a metal ion (11).

\section{Comparison with artificial aptamers}

Structural studies of many in vitro selected aptamer RNAs [reviewed in (32)] predate the discovery of small molecule-responsive natural riboswitches. These studies demonstrated the presence of structural motifs previously identified in natural RNAs in the artificial aptamers. For instance, structures of the ATP aptamer in complex with AMP (the aptamer does not recognize the phosphate groups) revealed that the adenosine binds to an internal loop of the aptamer that adopts the fold of the GNRA tetraloop (33), taking the place of the last $A$ of the motif and base pairing with the first $G$ of the motif in the manner of the natural loop (34). Several commonalities were suggested by analyses of a number of artificial aptamers including the use of stacking between base pairs and triples to recognize planar ligands and the coupling of aptamer folding with ligand binding (32). It was proposed that the latter is a defining characteristic of artificial aptamers, which evolved to satisfy a single function, ligand binding, unlike natural RNAs, which evolved as part of biological networks that require structural optimization. Comparison with structures of the aptamer domains of riboswitches confirms the wide-spread use of stacking and base pairing interactions in ligand recognition [compare, for instance, the structures of the FMN aptamer (35) and the FMN riboswitch (6)], and demonstrates a comparable degree of binding site-ligand shape complementarity in natural and artificial RNAs (36), but refutes the notion that ligand binding-induced folding (global induced fit) is a defining characteristic of in vitro evolved aptamers.

To a first approximation, it appears that the degree of ligand binding-induced folding of RNAs correlates inversely with their complexity. The preQ $_{1}$ riboswitch aptamer domain, the smallest known from nature (34 nt) folds only in the presence of ligand (37). In vitro selection experiments for high-affinity GTP aptamers resulted in a number of isolates that are more informationally complex than the lower-affinity ATP aptamer of similar global fold, suggesting that a structure capable of 10 -fold tighter binding requires specification of 
five additional nucleotides (38). Biochemical and structural analyses, however, demonstrate that these additional nucleotides do not make direct RNA-ligand interactions $(39,40)$. Rather, they engage in RNA-RNA interactions that pre-organize the RNA, reducing its conformational heterogeneity and increasing its propensity (41) to fold. Thus, most of the difference in affinity between a low affinity ATP aptamer and a high affinity GTP aptamer can be accounted for by a 45 -fold faster $k_{\text {on }}(40)$. The artificial RNA with the highest known affinity for its small molecule ligand $\left(K_{\mathrm{d}} \sim 0.8 \mathrm{nM}\right)$ is the $70 \mathrm{nt}$ tetracycline aptamer (42) (Table I). Biochemical and structural analysis of this Y-shaped RNA indicates that, unlike simple aptamers that are unfolded in the absence of ligand, this molecule is partially preorganized (42-44). Tetracycline is bound at a pocket formed between a 11-nt loop and the minor groove of an irregular duplex, and while the former appears to fold concomitantly with binding, the latter is structured in the absence of ligand (44). Such behavior is reminiscent of that of natural riboswitch aptamer domains of similar complexity. For instance, crystallographic (20), spectroscopic (45), calorimetric (28) and SAXS (46) analyses of the TPP riboswitch aptamer domain $(83 \mathrm{nt})$ indicate that ligand binding induces local as well as global conformational rearrangement, and that divalent cations facilitate ligand binding by pre-organizing the RNA, in addition to forming part of the TPP-RNA interface. The $g \operatorname{lm} S$ and lysine riboswitches, which have two of the largest riboswitch aptamer domains so far characterized ( $\sim 150$ and $\sim 180 \mathrm{nt}$, respectively) have global conformations that are insensitive to ligand occupancy $(7,9,10,47-49)$.

\section{Small molecule recognition by natural and artificial ribozymes}

\section{Diversity of ribozyme catalyzed reactions}

Thus far, natural ribozymes have been discovered that catalyze peptidyl transfer (the ribosome) and phosphoryl transfer reactions [reviewed in (50)]. The latter includes sequence-specific cleavage and ligation catalyzed by ribozymes through two types of reactions. The first is an internal transesterification involving the nucleophilic attack of a $2^{\prime}$ oxygen of a ribose on the adjacent $3^{\prime}$ phosphate group, resulting in two cleavage products, one containing a 2',3'-cyclic phosphate and the other a 5'-OH. Five classes of natural RNAs, known as the 'small self-cleaving ribozymes', are known to catalyze this reaction: the hammerhead, hairpin, hepatitis delta virus (HDV), Varkud satellite (VS), and $\operatorname{glm} S$ ribozymes [reviewed in (24)]. The second type of reaction is catalyzed by RNase P and the group I and II self-splicing introns (which are responsible for both phosphodiester cleavage and ligation), and related RNAs. These ribozymes yield either ligated exons or products with 5'-phosphate and 3'-OH termini, and can employ internal or external nucleophiles (50).

While the ability of RNAs to function as biochemical catalysts was first discovered in natural ribozymes, it has been through in vitro selections from randomized libraries by which most RNAs catalyzing different chemical transformations have been discovered. Artificial ribozymes include aminoacyl-RNA synthetases, RNA ligases and kinases ribozymes, nucleotide synthetases, thioester synthetases, and Diels-Alderases [reviewed in (51)]. Although few of these in vitro evolved ribozymes achieve rate enhancements comparable to those of natural ribozymes (52), their discovery has greatly broadened the known spectrum of ribozyme catalysis.

\section{Small molecule recognition by natural ribozymes}

Structural information is available on small molecule binding by two classes of natural ribozymes: the $g \operatorname{lm} S$ riboswitch and group I introns. The $g \operatorname{lm} S$ riboswitch is also a catalytic RNA. Binding of GlcN6P activates a latent self-cleavage activity of the ribozyme, increasing the rate of cleavage of a specific phosphodiester bond by $\sim 10^{6}$. GlcN6P functions as a coenzyme, and is absolutely required for activity of the ribozyme. Structures of $g \operatorname{lm} S$ 
riboswitches from two different bacterial species have been determined, each in multiple functional states [reviewed in (53)]. These studies suggest that neither binding of GlcN6P, catalytic activation, or product release is accompanied by RNA conformational changes. Indeed, the ribozyme is competent for GlcN6P binding and catalysis in the crystalline state. Thus, like other natural ribozymes [e.g. (54)], once the $g \operatorname{lmS}$ ribozyme folds, catalysis proceeds with motions localized to the active site. Binding of GlcN6P to the rigid glmS ribozyme is made possible by its open, solvent-accessible ligand binding site, which contrasts with the partially or totally solvent occluded binding sites of non-catalytic riboswitches. Analog studies show that the amine group of GlcN6P is essential for catalysis, and that the anomeric hydroxyl and phosphate groups of the coenzyme play important roles in binding $(55,56)$. The structures show that GlcN6P stacks underneath the nucleobase of the guanosine residue immediately following the scissile phosphate, with the phosphate of GlcN6P hydrogen bonding to N1 of the guanine. Outer-sphere coordinated divalent cations provide non-specific stabilization of GlcN6P binding. The amine of GlcN6P hydrogen bonds to the 5'-oxygen (the leaving group of the transesterification reaction), and the anomeric hydroxyl hydrogen bonds to the scissile phosphate. Experimental data are consistent with the amine of GlcN6P functioning as a general acid and electrostatic catalyst (57).

Group I introns employ the $3^{\prime}-\mathrm{OH}$ of an exogenous guanosine (exoG) as the nucleophile in the first transesterification reaction, resulting in release of the $5^{\prime}$ exon and addition exoG to the $5^{\prime}$ terminus of the intron. This is followed by local rearrangements of the ribozyme and replacement of exoG in the binding site with the conserved guanosine residue at the $3^{\prime}$ terminus of the intron $(\omega \mathrm{G})$, which provides the nucleophile for the second step of splicing. Structures have been determined of group I introns from three species in multiple functional states. Although none of these carry exoG, its mode of binding can be inferred from the manner in which $\omega \mathrm{G}$ is bound. The guanosine forms part of a base triple, and this triple is in turn sandwiched by two additional triples (Figure 4A). Although the participation of divalent cations in formation of the active site (including bridging ribose moieties of the guanosine with the substrate portion of the RNA) and in the catalytic steps is well established, the precise location of these ions in the transition state is a subject of active research. Different of lines of evidence indicate that exoG binding, release, and replacement with $\omega \mathrm{G}$ are accompanied by conformational rearrangements of the intron, but the structural details of these remain to be established because none of the currently available structures are determined at better than $3 \AA$ resolution [reviewed in (58)].

\section{Small molecule recognition by artificial ribozymes}

Structural information is available on small molecule binding by two classes of artificial ribozymes: a Diels-Alderase and flexizyme. The Diels-Alder reaction is a [4+2] cycloaddition of great importance in organic chemistry. Natural protein enzymes are known that catalyze this reaction, and Diels-Alderase catalytic antibodies have been selected by using transition state-mimics as haptens. Several in vitro selection experiments have been carried out to isolate Diels-Alderase ribozymes [reviewed in (59)]. The structure of a 49-nt Diels-Alderase selected to catalyze the cycloaddition of a biotinylated maleimide and a tethered anthracene derivative (60) was determined both free and bound to the reaction product (61). The RNA folds into a compact double pseudoknot that cradles the cycloaddition product in a solvent-exposed groove, interacting with it primarily through aromatic-aromatic stacking and non-polar van der Waals contacts, using very limited hydrogen bonding. Shape complementarity of the ribozyme to the product is good, but calculations suggest that it is even better to the transition state. Thus, catalysis appears to result from proximity effects (binding and positioning of the reactants) and preferential 
transition state binding. The rigidity of the folded ribozyme ensures a high degree of regioselectivity [see also (62)].

Flexizyme catalyzes the regioselective 3 '-aminoacylation of tRNAs using pre-activated (e.g. adenylated) amino acids (63). The 45-nt flexizyme recognizes its tRNA substrate by base pairing to its NCCA 3'-terminus (64), and positions the acceptor helix approximately perpendicular to an irregular, underwound active site helix. The minor groove of this helix buckles open to expose the planar faces of two nucleotides to provide a binding site for the amino acid side chain. The nature of this pocket is consistent with flexizyme's strong preference for aromatic amino acids. Indeed, cocrystallization of flexizyme with phenylalanine-ethylester (an inhibitor) suggests that the benzyl moiety stacks on G24 so as to maximize polar-aromatic interactions (65). This positions the carbonyl of the amino acid in van der Waals contact with the nucleophile 3'-OH of the tRNA (Figure 4B). Structural and biochemical analyses suggest that flexizyme can achieve considerable specificity despite this simple, open active site architecture by coupling amino acid binding to productive positioning of the acceptor terminus, that is, employing an induced-fit mechanism.

\section{Small molecule recognition by natural and artificial ribozymes}

Although the basic small-molecule recognition strategies employed by ribozymes (such as stacking, base pairing and cation-mediated binding) are the same as those of riboswitches, ribozyme active sites appear to be qualitatively simpler than riboswitch ligand binding pockets. This in part must reflect the fact that crystal structures are of ground-state complexes, while ribozyme active sites have evolved to be complementary to the transition state (66). The $g \operatorname{lm} S$ riboswitch and the Diels-Alderase appear to be rigid structures that permit catalysis to proceed with minimal additional loss of entropy once the substrates (cofactor, for $g \operatorname{lm} S$ ) are bound. On the other hand, group I intron active sites necessarily must remain flexible so that the same active site can catalyze both steps of splicing. It appears that flexizyme has evolved to exploit its flexibility to couple amino acid binding site structure formation to productive tRNA docking, and in this way overcomes the limitations of its rather featureless amino acid binding pocket.

\section{Two paths to the same outcome: kinetic and thermodynamic control of riboswitches}

\section{Diverse mechanisms of genetic control}

How do the conformational and energetic changes undergone by natural or artificial RNAs upon ligand binding result in alteration of gene expression? The tetracycline aptamer has been employed as an artificial riboswitch to modulate alternative pre-mRNA splicing (67) or translation initiation (68). In Gram-positive bacteria the GlcN6P-dependent self-cleaving activity of the $g \operatorname{lm} S$ ribozyme controls the stability of the mRNA it is part of (69). The only riboswitch class thus far discovered in eukaryotes is the TPP riboswitch. In plants, fungi and algae, these riboswitches control alternative splicing. In all characterized cases, segments of the aptamer domain of the ligand-free TPP riboswitch (such as the pyrophosphate binding nucleotides) can base pair with nearby splice sites, occluding them. Upon TPP binding, the riboswitch aptamer domain folds, and exposes the previously occluded splice site, leading to changes in the pre-mRNA splicing pattern [reviewed in (70)]. In bacteria, most riboswitches appear to function by transcription attenuation (termination versus readthrough) or translation initiation control. For these mechanisms, sequence elements in addition to the riboswitch aptamer domain are needed. These have been termed the 'expression platform' (3). Transcription attenuation, particularly effective in Gram-positive bacteria due to increased operon size and complexity, primarily makes use of an overlapping RNA segment, 
which chooses between mutually exclusive RNA structures leading to either premature transcription termination or readthrough.

Transduction of ligand binding into a genetic decision can occur under kinetic or thermodynamic control. The relevant parameters can include the rate of aptamer folding, ligand concentration, $k_{\mathrm{on}}, k_{\mathrm{off}}$, and $K_{\mathrm{d}}$ for aptamer, speed of transcription, delay time at transcriptional pauses, competition between alternative RNA structures, height of activation barrier between bistable RNA structures, etc. A kinetic control scheme precisely gears the kinetic parameters of transcription to those of ligand binding and co-transcriptional folding of aptamers, whereas a thermodynamic scheme depends on an equilibrium between ligand association and dissociation, or between bistable, inter-converting RNA structures whose relative populations are shifted by the concentration of ligands.

A large discrepancy between the concentration of FMN required to elicit a half maximal shift in termination efficiency in vitro $\left(T_{50}\right)$ and the dissociation constant prompted two studies of the kinetic basis of FMN and adenine riboswitch regulation $(71,72)$. By monitoring the binding- induced quenching of FMN and 2-amino-purine fluorescence, $k_{\mathrm{on}}$ and $k_{\text {off }}$ values were directly measured in these studies. [Interestingly, most riboswitch aptamers examined to date exhibit similar $k_{\text {on }}$ values on the order of $10^{4}$ to $10^{5} \mathrm{M}^{-1} \mathrm{~s}^{-1}$, whereas $k_{\text {off }}$ are found to range from $10^{-3}$ to $10^{-1} \mathrm{~s}^{-1}$ (Table 1).] These quantities, combined with commonly observed micromolar levels of metabolite concentrations, place the time constant $\tau\left(\sim 1 / k_{\text {off }}\right)$ leading to association and dissociation equilibria in the range of seconds. This is comparable to that of the elongation of RNA polymerase $\left(\Delta t_{\text {RNAP }}\right)$ from the end of the aptamer domain to the termination decision point. $\Delta t_{\text {RNAP }}$ depends on the length of the expression platform, the elongation speed of the cognate RNAP, and importantly, the recognition efficiency and dwell times at any long-lived transcriptional pause sites (73).

The hallmark of kinetic control is $\Delta \mathrm{t}_{\mathrm{RNAP}}<\tau$, which means that RNAP arrives at the termination decision point before ligand and aptamer reach binding equilibrium (Figure 5 , $\tau_{2}$, green dot). Since equilibrium is not reached, a ligand concentration equivalent to $K_{\mathrm{d}}$ does not lead to 50\% ligand binding, producing significant discrepancies between $T_{50}$ and $K_{\mathrm{d}}$. In contrast, a thermodynamic mechanism would need to satisfy $\Delta \mathrm{t}_{\mathrm{RNAP}}>\tau$, which allows ligand and aptamer to reach equilibrium before RNAP reaches the transcription terminator (Figure $5, \tau_{1}$, orange dot). Under these conditions, $T_{50}$ would approximate $K_{\mathrm{d}}$. Conceivably, the choice of kinetic or thermodynamic control may have evolved to match the range of $K_{\mathrm{d}}$ and the intracellular level of ligand in question. It is also possible for a riboswitch to operate under either kinetic or thermodynamic control depending on environmental cues such as nutritional availability that would alter intracellular NTP levels, and therefore elongation rates and pause durations.

\section{Kinetic control: the FMN riboswitch}

FMN binding to the B. subtilis ribD FMN riboswitch aptamer occurs with $k_{\text {on }}=2.0 \times 105$ $\mathrm{M}^{-1} \mathrm{~s}^{-1}, k_{\text {off }}=0.002 \mathrm{~s}-1, K_{\mathrm{d}}=12 \mathrm{nM}$, and $T_{50}=200-500 \mathrm{nM}$. A $T_{50}$ much higher than $K_{\mathrm{d}}$ suggests kinetic control in which substantially more FMN is required rapidly to occupy $50 \%$ of the aptamers before the RNAP reaches the termination decision point. As expected for a kinetically regulated riboswitch, the termination efficiency is sensitive to NTP concentrations. Two prominent transcriptional pauses were identified that when disrupted led to decreased termination and another 4-fold increase in $T_{50}$. This suggests that these functionally important, long-lived pauses provide necessary delays for FMN to bind to the aptamer (71). 


\section{Thermodynamic control: the $\mathrm{preQ}_{1}$ riboswitch}

Fluorescence and NMR spectroscopic studies of the $F$. nucleatum preQ $_{1}$ riboswitch revealed the co-existence of two competing stem-loop structures in the expression platform, corresponding to the antiterminator and terminator hairpins. The relative populations of these two structures at equilibrium are rapidly (relative to elongation rates of some bacterial RNAPs) shifted by the presence of preQ ${ }_{1}$, allowing for thermodynamic control. However, unavailability of cognate $F$. nucleatum RNAP precluded accurate estimates of $\Delta \mathrm{t}_{\mathrm{RNAP}}$, and thus definitive determination of the control mechanism for this riboswitch (37). Nonetheless, the bistable nature of the terminator and antiterminator hairpin loops and their rapid exchange suggests a paradigm for riboswitches under thermodynamic control.

\section{From simplified models to realistic regulatory scenarios Nascent RNA structures impact transcription}

Riboswitch and ribozyme function in vivo can be much more complex than in these defined in vitro systems, due to the rugged kinetic landscape of RNAP elongation through these structured RNAs, co-transcriptional folding of aptamers, modulation of RNA folding rates by cognate and non-cognate small molecules, coupling of translation with transcription, and the action of RNA binding proteins. During the course of transcription, nascent RNA structures such as pause hairpins exert significant effects on the kinetics of transcription $(74,75)$. Some nascent RNAs can bind the RNAP and confer to it robust anti-pausing and anti-termination capabilities. Examples are the PutL RNA in the lambdoid phage HK22 antitermination (76), and the B. subtilis EAR (eps-associated RNA) element, which was proposed to associate with RNAP and confer processivity necessary to complete an usually long (16kb) eps (exopolysaccharide) transcript important in biofilm formation (77). The effects of ligand binding (or $\mathrm{glmS}$ riboswitch self cleavage or intron self-splicing) on nascent riboswitch transcription (including RNAP pausing) remains to be elucidated.

\section{Kinetic coupling and regulator trafficking between translation and transcription machines}

In bacteria, trailing ribosomes typically travel slower than leading RNAP, and thus also utilize transcriptional pauses to ensure coupling of translation to transcription, preventing the termination factor Rho from loading onto naked RNA and causing undesired termination. Ribosomes not only drive paused RNAPs back to elongation by disrupting pause hairpins but also directly modulate the rates of transcription by preventing spontaneous RNAP backtracking and providing assistance through DNA roadblocks (78). How ligand binding to riboswitches affects this coupling is unknown. Moreover, little kinetic characterization of riboswitches that function through translation has been reported.

\section{RNA binding proteins and chaperones may affect riboswitch function}

In addition to being restricted by the leading RNAP and trailing ribosome, the riboswitch mRNA emerging from the RNAP exit channel is subject to binding and potential control by additional protein factors. NusA, docked on the RNAP exit channel during elongation, may hand over partially folded nascent RNA structures onto other protein factors such as ribosomal protein L7Ae homologs (e.g. YbxF and Yl×q in B. subtilis), which recognize Kturn motifs present in many structured RNAs (e.g., SAM-I, Lysine, and T-box riboswitches) (79-81). While riboswitches have been shown to function without proteins in vitro, RNAbinding proteins may play architectural roles by assisting folding of complex aptamers, regulatory roles by selectively stabilizing one of mutually exclusive RNA structures, introducing post-transcriptional RNA modifications (82), or even directly acting as the signaling ligand, such as the case of L4 associating with the S10 operon 5' leader and causing termination of its own operon (83). 
Riboswitches and ribozymes provide a biological context where small molecule recognition by RNA, RNA folding assisted by small molecules, and transcription, translation, and splicing kinetics influenced by nascent RNA structures synergistically operate genetic switches. In light of the rapid pace of structure determination of natural and artificial riboswitches and ribozymes, the interface between the RNA-ligand modules and their expression platforms warrants more research. A mechanistic understanding of such coupling would allow modular design and rational engineering of artificial bacterial and mammalian riboswitches and ribozymes as well as modulation of their natural counterparts.

\section{Acknowledgments}

We thank N. Baird and S. Woodson for discussions.

\section{Abbreviations}

$\begin{array}{ll}\text { c-di-GMP } & \text { cyclic diguanylate } \\ \text { EAR } & \text { eps-associated RNA } \\ \text { exoG } & \text { exogenous guanosine } \\ \text { FMN } & \text { flavin mononucleotide } \\ \text { GIcN6P } & \text { glucosamine-6-phosphate } \\ \text { GTP } & \text { guanosine triphosphate } \\ \text { HDV } & \text { hepatitis delta virus } \\ \text { NTP } & \text { nucleotide triphosphate } \\ \text { WG } & \text { the conserved guanosine at the 3' terminus of Group I introns } \\ \text { RNAP } & \text { RNA polymerase } \\ \text { SAM } & \text { S-adenosylmethionine } \\ \text { SAXS } & \text { small angle X-ray scattering } \\ \text { TMP } & \text { thiamine monophosphate } \\ \text { TPP } & \text { thiamine pyrophosphate } \\ \text { VS } & \text { Varkud satellite }\end{array}$

\section{References}

1. Loh E, Dussurget O, Gripenland J, Vaitkevicius K, Tiensuu T, Mandin P, Repoila F, Buchrieser C, Cossart P, Johansson J. A trans-acting riboswitch controls expression of the virulence regulator PrfA in Listeria monocytogenes. Cell. 2010; 139:770-779. [PubMed: 19914169]

2. Rajkowitsch L, Chen D, Stampfl S, Semrad K, Waldsich C, Mayer O, Jantsch MF, Konrat R, Bläsi U, Schroeder R. RNA chaperones, RNA annealers and RNA helicases. RNA Biology. 2007; 4:118130. [PubMed: 18347437]

3. Winkler W, Nahvi A, Breaker RR. Thiamine derivatives bind messenger RNAs directly to regulate bacterial gene expression. Nature. 2002; 419:952-956. [PubMed: 12410317]

4. Kulshina N, Baird NJ, Ferré-D'Amaré AR. Recognition of the bacterial second messenger cyclic diguanylate by its cognate riboswitch. Nat Struct Mol Biol. 2009; 16:1212-1217. [PubMed: 19898478]

5. Smith KD, Lipchock SV, Ames TD, Wang J, Breaker RR, Strobel SA. Structural basis of ligand binding by a c-di-GMP riboswitch. Nat Struct Mol Biol. 2009; 16:1218-1223. [PubMed: 19898477] 
6. Serganov A, Huang L, Patel D. Coenzyme recognition and gene regulation by a flavin mononucleotide riboswitch. Nature. 2009; 457:233-237. [PubMed: 19169240]

7. Klein DJ, Ferré-D'Amaré AR. Structural basis of $g \operatorname{lm} S$ ribozyme activation by glucosamine-6phosphate. Science. 2006; 313:1752-1756. [PubMed: 16990543]

8. Cochrane JC, Lipchock SV, Strobel SA. Structural investigation of the $g \operatorname{lmS}$ ribozyme bound to its catalytic cofactor. Chem Biol. 2007; 14:97-105. [PubMed: 17196404]

9. Serganov A, Huang L, Patel D. Structural insights into amino acid binding and gene control by a lysine riboswitch. Nature. 2008; 455:1263-1267. [PubMed: 18784651]

10. Garst AD, Héroux A, Rambo RP, Batey RT. Crystal structure of the lysine riboswitch regulatory mRNA element. J Biol Chem. 2008; 283:22347-22351. [PubMed: 18593706]

11. Klein D, Edwards T, Ferré-D'Amaré A. Cocrystal structure of a class I preQ ${ }_{1}$ riboswitch reveals a pseudoknot recognizing an essential hypermodified nucleobase. Nat Struct Mol Biol. 2009; 16:343-344. [PubMed: 19234468]

12. Kang M, Peterson R, Feigon J. Structural insights into riboswitch control of the biosynthesis of queuosine, a modified nucleotide found in the anticodon of tRNA. Mol Cell. 2010; 39:653-655.

13. Spitale R, Torelli A, Krucinska J, Bandarian V, Wedekind J. The structural basis for recognition of the preQ $_{0}$ metabolite by an unusually small riboswitch aptamer domain. J Biol Chem. 2009; 284:11012-11016. [PubMed: 19261617]

14. Dann, Wakeman; Sieling, Baker; Irnov; Winkler. Structure and mechanism of a metal-sensing regulatory RNA. Cell. 2007; 130:878-892. [PubMed: 17803910]

15. Batey RT, Gilbert SD, Montange RK. Structure of a natural guanine-responsive riboswitch complexed with the metabolite hypoxanthine. Nature. 2004; 432:411-415. [PubMed: 15549109]

16. Serganov A, Yuan YR, Pikovskaya O, Polonskaia A, Malinina L, Phan AT, Hobartner C, Micura R, Breaker RR, Patel DJ. Structural basis for discriminative regulation of gene expression by adenine- and guanine-sensing mRNAs. Chem Biol. 2004; 11:1729-1741. [PubMed: 15610857]

17. Montange RK, Batey RT. Structure of the S-adenosylmethionine riboswitch regulatory mRNA element. Nature. 2006; 441:1172-1175. [PubMed: 16810258]

18. Gilbert SD, Rambo RP, Van Tyne D, Batey RT. Structure of the SAM-II riboswitch bound to Sadenosylmethionine. Nat Struct Mol Biol. 2008; 15:177-182. [PubMed: 18204466]

19. Lu C, Smith AM, Fuchs RT, Ding F, Rajashankar K, Henkin TM, Ke A. Crystal structures of the SAM-III/SMK riboswitch reveal the SAM-dependent translation inhibition mechanism. Nat Struct Mol Biol. 2008; 15:1076-1083. [PubMed: 18806797]

20. Edwards TE, Ferré-D'Amaré AR. Crystal structures of the thi-box riboswitch bound to thiamine pyrophosphate analogs reveal adaptive RNA-small molecule recognition. Structure. 2006; 14:1459-1468. [PubMed: 16962976]

21. Serganov A, Polonskaia A, Phan AT, Breaker RR, Patel DJ. Structural basis for gene regulation by a thiamine pyrophosphate-sensing riboswitch. Nature. 2006; 441:1167-1171. [PubMed: 16728979]

22. Thore S. Structure of the eukaryotic thiamine pyrophosphate riboswitch with its regulatory ligand. Science. 2006; 312:1208-1211. [PubMed: 16675665]

23. Lilley, DMJ.; Eckstein, F. Ribozymes and RNA catalysis: introduction and primer. In: Lilley, DMJ.; Eckstein, F., editors. Ribozymes and RNA catalysis. The Royal Society of Chemistry; Cambridge: 2008. p. 1-10.

24. Ferré-D'Amaré, AR.; Scott, WG. Cold Spring Harbor Perpectives Biol. 2010. Small self-cleaving ribozymes. (published online September 15)

25. Pleij CWA, Rietveld K, Bosch L. A new principle of RNA folding based on pseudoknotting. Nucleic Acids Res. 1985; 13:1717-1731. [PubMed: 4000943]

26. de la Peña M, Dufour D, Gallego J. Three-way RNA junctions with remote tertiary contacts: A recurrent and highly versatile fold. RNA. 2009; 15:1949-1964. [PubMed: 19741022]

27. Kondo J, Westhof E. Base pairs and pseudo pairs observed in RNA-ligand complexes. J Mol Recognit. 2009; 23:241-252. [PubMed: 19701919] 
28. Kulshina N, Edwards TE, Ferré-D'Amaré AR. Thermodynamic analysis of ligand binding and ligand binding-induced tertiary structure formation by the thiamine pyrophosphate riboswitch. RNA. 2010; 16:186-196. [PubMed: 19948769]

29. Gilbert SD, Stoddard CD, Wise SJ, Batey RT. Thermodynamic and kinetic characterization of lignd binding to the purine riboswitch aptamer domain. J Mol Biol. 2006; 359:754-768. [PubMed: 16650860]

30. Mandal M, Boese B, Barrick JE, Winkler WC, Breaker RR. Riboswitches control fundamental biochemical pathways in Bacillus subtilis and other bacteria. Cell. 2003; 113:577-586. [PubMed: 12787499]

31. Roth, Winkler; Regulski, Lee; Lim, Jona; Barrick, Ritwik; Kim, Welz; Iwata-Reuyl; Breaker. A riboswitch selective for the queuosine precursor preQ $Q_{1}$ contains an unusually small aptamer domain. Nat Struct Mol Biol. 2007; 14:308-317. [PubMed: 17384645]

32. Hermann T, Patel DJ. Adaptive recognition by nucleic acid aptamers. Science. 2000; 287:820-825. [PubMed: 10657289]

33. Jucker FM, Pardi A. GNRA tetraloops make a U-turn. RNA. 1995; 1:219-222. [PubMed: 7585251]

34. Jiang F, Kumar RA, Jones RA, Patel DJ. Structural basis of RNA folding and recognition in an AMP-RNA aptamer complex. Nature. 1996; 382:183-186. [PubMed: 8700212]

35. Fan P, Suri AK, Fiala R, Live D, Patel DJ. Molecular recognition in the FMN-RNA aptamer complex. J Mol Biol. 1996; 258:480-500. [PubMed: 8642604]

36. Edwards TE, Klein DJ, Ferré-D'Amaré AR. Riboswitches: small-molecule recognition by gene regulatory RNAs. Curr Op Struct Biol. 2007; 17:273-279.

37. Rieder U, Kreutz C, Micura R. Folding of a transcriptionally acting preQ 1 riboswitch. Proc Natl Acad Sci USA. 2010; 107:10804-10809. [PubMed: 20534493]

38. Carothers JM, Oestreich SC, Davis JH, Szostak JW. Informational complexity and functional activity of RNA structures. J Am Chem Soc. 2004; 126:5130-5137. [PubMed: 15099096]

39. Carothers J, Oestreich S, Szostak J. Aptamers selected for higher-affinity binding are not more specific for the target ligand. J Am Chem Soc. 2006; 128:7929-7937. [PubMed: 16771507]

40. Carothers JM, Davis JH, Chou JJ, Szostak JW. Solution structure of an informationally complex high-affinity RNA aptamer to GTP. RNA. 2006; 12:567-579. [PubMed: 16510427]

41. Schultes EA, Spasic A, Mohanty U, Bartel DP. Compact and ordered collapse of randomly generated RNA sequences. Nat Struct Mol Biol. 2005; 12:1130-1136. [PubMed: 16273104]

42. Müller M, Weigand JE, Weichenrieder O, Suess B. Thermodynamic characterization of an engineered tetracycline-binding riboswitch. Nucleic Acids Res. 2006; 34:2607-2617. [PubMed: 16707663]

43. Hanson S, Bauer G, Fink B, Suess B. Molecular analysis of a synthetic tetracycline-binding riboswitch. RNA. 2005; 11:503-511. [PubMed: 15769877]

44. Xiao H, Edwards TE, Ferré-D'Amaré AR. Structural basis for specific, high-affinity tetracycline binding by an in vitro evolved aptamer and artificial riboswitch. Chem Biol. 2008; 15:1125-1137. [PubMed: 18940672]

45. Lang K, Rieder R, Micura R. Ligand-induced folding of the thiM TPP riboswitch investigated by a structure-based fluorescence spectroscopic approach. Nucleic Acids Res. 2007; 35:5370-5378. [PubMed: 17693433]

46. Baird NJ, Kulshina N, Ferré-D'Amaré AR. Riboswitch function: flipping the switch or tuning the dimmer? RNA Biol. 2010; 7:328-332. [PubMed: 20458165]

47. Baird NJ, Ferré-D'Amaré AR. Idiosyncratically tuned switching behavior of riboswitch aptamer domains revealed by comparative small-angle X-ray scattering analysis. RNA. 2010; 16:598-609. [PubMed: 20106958]

48. Hampel KJ, Tinsley MM. Evidence for preorganization of the $\mathrm{glmS}$ ribozyme ligand binding pocket. Biochemistry. 2006; 45:7861-7871. [PubMed: 16784238]

49. Tinsley RA, Furchak JR, Walter NG. Trans-acting glmS catalytic riboswitch: locked and loaded. RNA. 2007; 13:468-477. [PubMed: 17283212] 
50. Fedor MJ, Williamson JR. The catalytic diversity of RNAs. Nat Rev Mol Cell Biol. 2005; 6:399412. [PubMed: 15956979]

51. Chen X, Li N, Ellington AD. Ribozyme catalysis of metabolism in the RNA world. Chem Biodivers. 2007; 4:633-655. [PubMed: 17443876]

52. Silverman, SK. Artificial functional nucleic acids: aptamers ribozymes and deoxyribozymes identified by in vitro selection. In: Li, Y.; Lu, Y., editors. Functional Nucleic Acids for Analytical Applications. Springer; New York: 2009. p. 2-76.

53. Ferré-D'Amaré, AR. Q Rev Biophys. 2010. The $g \operatorname{lm} S$ ribozyme: use of a small molecule coenzyme by a gene-regulatory RNA. (published online September 8)

54. Ferré-D'Amaré AR. The hairpin ribozyme. Biopolymers. 2004; 73:71-78. [PubMed: 14691941]

55. McCarthy TJ, Plog MA, Floy SA, Jansen JA, Soukup JK, Soukup GA. Ligand requirements for glmS ribozyme self-cleavage. Chem Biol. 2005; 12:1221-1226. [PubMed: 16298301]

56. Klein DJ, Wilkinson SR, Been MD, Ferré-D'Amaré AR. Requirement of helix P2.2 and nucleotide G1 for positioning of the cleavage site and cofactor of the $g \operatorname{lmS}$ ribozyme. J Mol Biol. 2007; 373:178-189. [PubMed: 17804015]

57. Klein DJ, Been MD, Ferré-D'Amaré AR. Essential role of an active-site guanine in glmS ribozyme catalysis. J Am Chem Soc. 2007; 129:14858-14859. [PubMed: 17990888]

58. Woodson SA. Structure and assembly of group I introns. Curr Opin Struct Biol. 2005; 15:324-330. [PubMed: 15922592]

59. Pitt JN, Ferré-D'Amaré AR. How RNA closes a Diel. Nat Struct Mol Biol. 2005; 12:206-208. [PubMed: 15744318]

60. Seelig B, Jaschke A. A small catalytic RNA motif with Diels-Alderase activity. Chem Biol. 1999; 6:167-176. [PubMed: 10074465]

61. Serganov A, Keiper S, Malinina L, Tereshko V, Skripkin E, Hobartner C, Polonskaia A, Tuan Phan A, Wombacher R, Micura R, Dauter Z, Jaschke A, Patel DJ. Structural basis for Diels-Alder ribozyme catalyzed carbon-carbon bond formation. Nature Struct Mol Biol. 2005; 12:218-224. [PubMed: 15723077]

62. Pitt J, Ferré-D'Amaré AR. Structure-guided engineering of the regioselectivity of RNA ligase ribozymes. J Am Chem Soc. 2009; 131:3532-3540. [PubMed: 19220054]

63. Saito H, Kourouklis D, Suga H. An in vitro evolved precursor tRNA with aminoacylation activity. EMBO J. 2001; 20:1797-1806. [PubMed: 11285242]

64. Bessho Y, Hodgson DRW, Suga H. A tRNA aminoacylation system for non-natural amino acids based on a programmable ribozyme. Nature Biotechnology. 2002; 20:723-728.

65. Xiao H, Murakami H, Suga H, Ferré-D'Amaré AR. Structural basis of specific tRNA aminoacylation by a small in vitro selected ribozyme. Nature. 2008; 454:358-361. [PubMed: 18548004]

66. Rupert PB, Massey AP, Sigurdsson ST, Ferré-D'Amaré AR. Transition state stabilization by a catalytic RNA. Science. 2002; 298:1421-1424. [PubMed: 12376595]

67. Weigand JE, Suess B. Tetracycline aptamer-controlled regulation of pre-mRNA splicing in yeast. Nucleic Acids Res. 2007; 35:4179-4185. [PubMed: 17567606]

68. Kotter P, Weigand JE, Meyer B, Entian KD, Suess B. A fast and efficient translational control system for conditional expression of yeast genes. Nucleic Acids Res. 2009; 37:e120-e120. [PubMed: 19592423]

69. Collins JA, Irnov I, Baker S, Winkler WC. Mechanism of mRNA destabilization by the glmS ribozyme. Genes Dev. 2007; 21:3356-3368. [PubMed: 18079181]

70. Wachter A. Riboswitch-mediated control of gene expression in eukaryotes. RNA Biology. 2010; 7:67-76. [PubMed: 20009507]

71. Wickiser JK, Winkler WC, Breaker RR, Crothers DM. The speed of RNA transcription and metabolite binding kinetics operate an FMN riboswitch. Mol Cell. 2005; 18:49-60. [PubMed: 15808508]

72. Wickiser JK, Cheah MT, Breaker RR, Crothers DM. The kinetics of ligand binding by an adeninesensing riboswitch. Biochemistry. 2005; 44:13404-13414. [PubMed: 16201765] 
73. Artsimovitch I, Landick R. Pausing by bacterial RNA polymerase is mediated by mechanistically distinct classes of signals. Proc Natl Acad Sci USA. 2000; 97:7090-7095. [PubMed: 10860976]

74. Toulokhonov I, Artsimovitch I, Landick R. Allosteric control of RNA polymerase by a site that contacts nascent RNA hairpins. Science. 2001; 292:730-733. [PubMed: 11326100]

75. Zhang J, Palangat M, Landick R. Role of the RNA polymerase trigger loop in catalysis and pausing. Nat Struct Mol Biol. 2010; 17:99-104. [PubMed: 19966797]

76. Komissarova N, Velikodvorskaya T, Sen R, King RA, Banik-Maiti S, Weisberg RA. Inhibition of a transcriptional pause by RNA anchoring to RNA polymerase. Mol Cell. 2008; 31:683-694. [PubMed: 18775328]

77. Irnov I, Winkler WC. A regulatory RNA required for antitermination of biofilm and capsular polysaccharide operons in Bacillales. Mol Microbiol. 2010; 76:559-575. [PubMed: 20374491]

78. Proshkin S, Rahmouni AR, Mironov A, Nudler E. Cooperation between translating ribosomes and RNA polymerase in transcription elongation. Science. 2010; 328:504-508. [PubMed: 20413502]

79. Green NJ, Grundy FJ, Henkin TM. The T box mechanism: tRNA as a regulatory molecule. FEBS Lett. 2010; 584:318-324. [PubMed: 19932103]

80. Grundy FJ, Henkin TM. tRNA as a positive regulator of transcription antitermination in B. subtilis. Cell. 1993; 74:475-482. [PubMed: 8348614]

81. Blouin S, Lafontaine DA. A loop loop interaction and a K-turn motif located in the lysine aptamer domain are important for the riboswitch gene regulation control. RNA. 2007; 13:1256-1267. [PubMed: 17585050]

82. Ferré-D'Amaré AR. RNA-modifying enzymes. Curr Op Struct Biol. 2003; 13:49-55.

83. Zengel JM, Lindahl L. Ribosomal protein L4 stimulates in vitro termination of transcription at a NusA-dependent terminator in the S10 operon leader. Proc Natl Acad Sci U S A. 1990; 87:26752679. [PubMed: 2157208]

84. Jucker FM, Phillips RM, McCallum SA, Pardi A. Role of a heterogeneous free state in the formation of a specific RNA-theophylline complex. Biochemistry. 2003; 42:2560-2567. [PubMed: 12614150]

85. Karbstein K, Herschlag D. Extraordinarily slow binding of guanosine to the Tetrahymena group I ribozyme: implications for RNA preorganization and function. Proc Natl Acad Sci USA. 2003;

100:2300-2305. [PubMed: 12591943] 

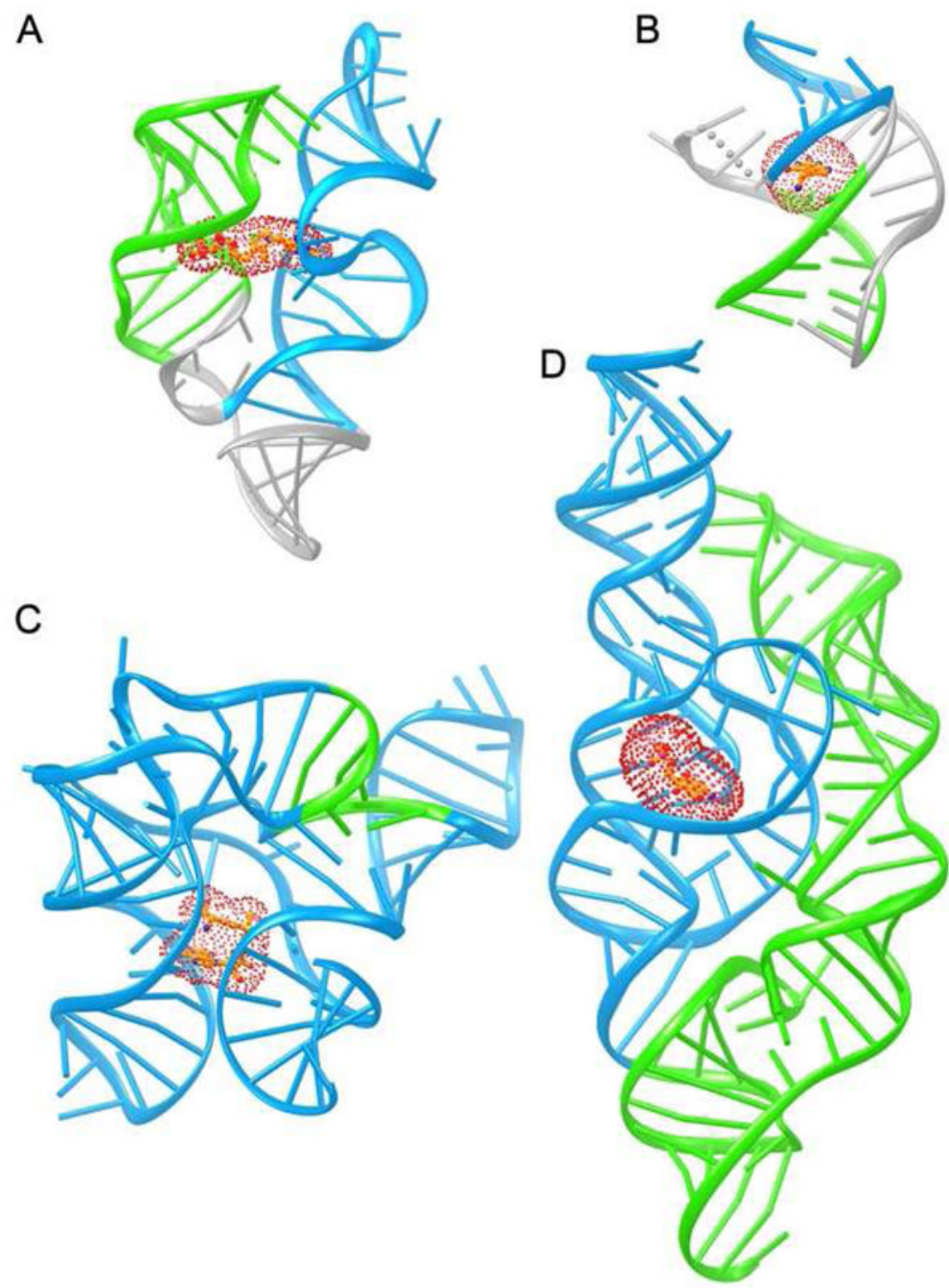

FIGURE 1.

Overall structure of representative riboswitch aptamer domains in cartoon form. (A) The TPP riboswitch (21) adopts a Y-shaped structure that recognizes its ligand (red) in an elongated conformation between the pyrimidine-binding helix (cyan) and the pyrophosphate binding helix (green). (B) The preQ $\mathrm{Q}_{1}$ riboswitch (11) folds as an H-type pseudoknot, with its ligand continuing the stack between the two A-form helices (cyan and green). (C) The SAM-I riboswitch (17) is organized around a four helix junction (cyan) but also comprises a pseudoknot (green). (D) The glmS ribozyme-riboswitch is comprised of a doublepseudoknot core domain (cyan) and a peripheral domain (green) that consists of a pseudoknot that functions as a 3-helix junction. 


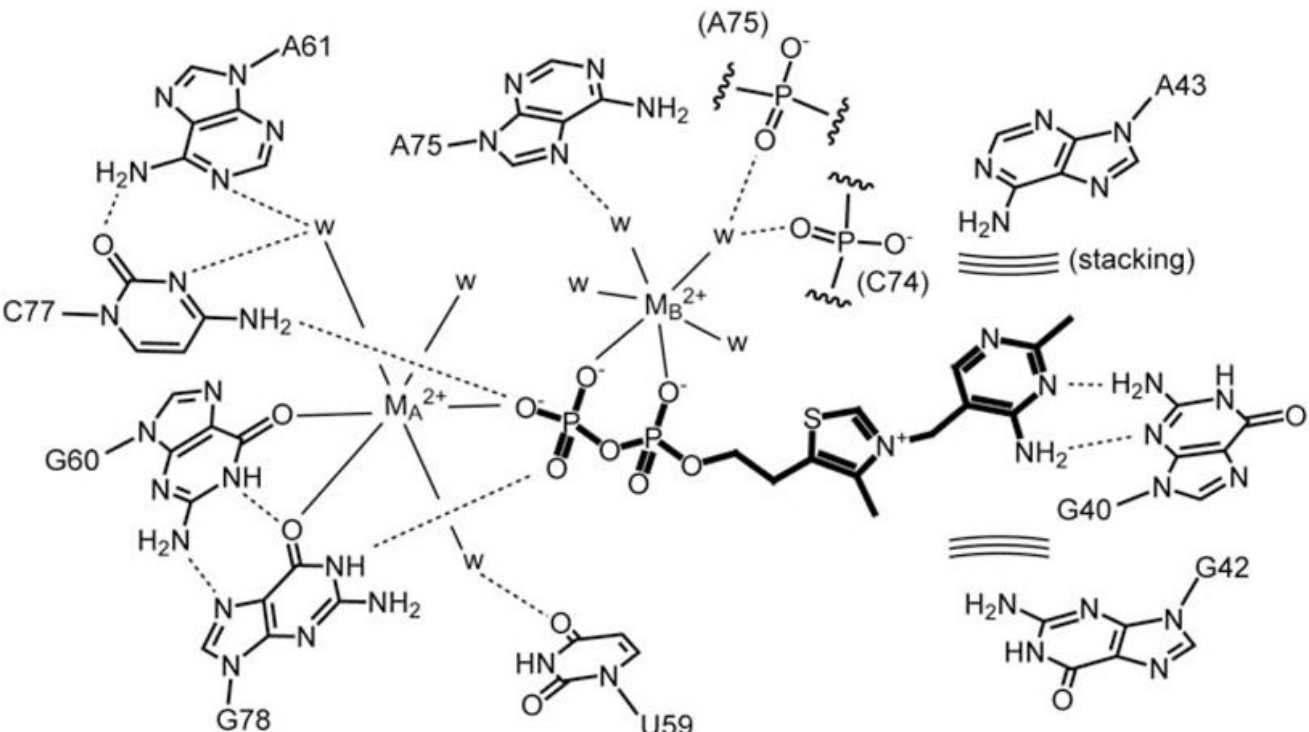

FIGURE 2.

Ligand recognition by the TPP riboswitch. The pyrimidine is recognized through a combination of base stacking and base pairing. The pyrophosphate is recognized primarily through two chelated divalent cations $\left(\mathrm{M}_{\mathrm{A}} 2+\right.$ and $\left.\mathrm{M}_{\mathrm{B}} 2+\right)$ and their hydration sphere. 
A

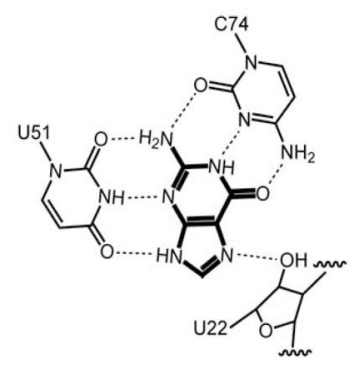

C

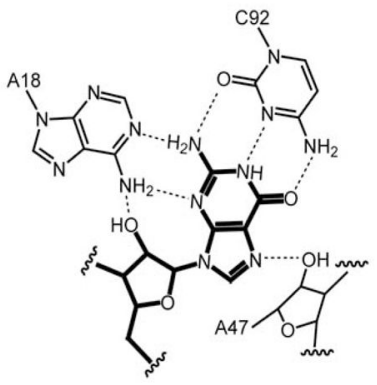

B

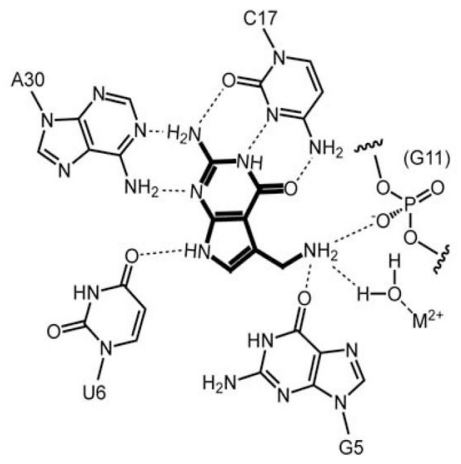

D

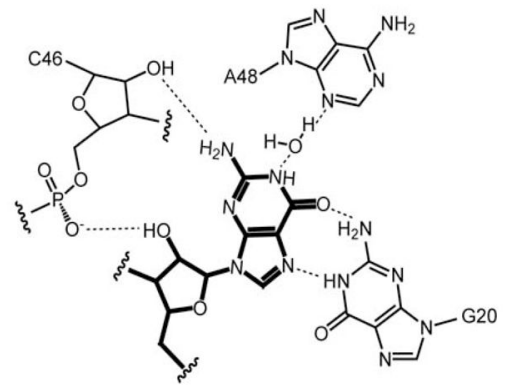

FIGURE 3.

Comparison of base-pairing schemes employed by three riboswitches that recognize guanine and related ligands. The four examples are the (A) purine, $(\mathrm{B}) \mathrm{preQ}_{1}$, and $(\mathrm{C})$ first $\left(\mathrm{g}_{\mathrm{I}}\right)$ and (D) second guanine $\left(\mathrm{g}_{\mathrm{II}}\right)$ residues of c-di-GMP bound to its cognate riboswitch. 
A
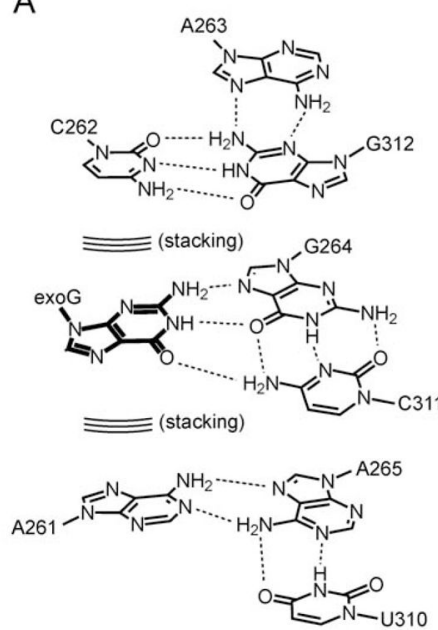

B

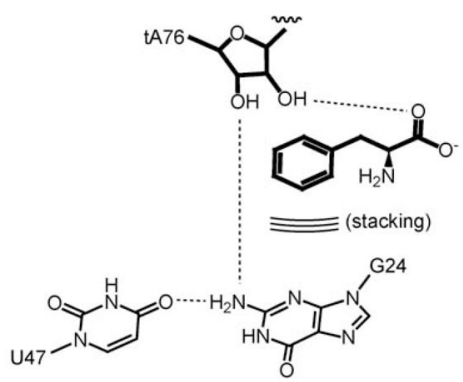

FIGURE 4.

Ligand recognition strategies employed by (A) the Tetrahymena group I intron in recognizing the base of the exogenous guanosine substrate, and (B) flexizyme in positioning the acceptor end (residue 76) of tRNA and phenylalanine. 


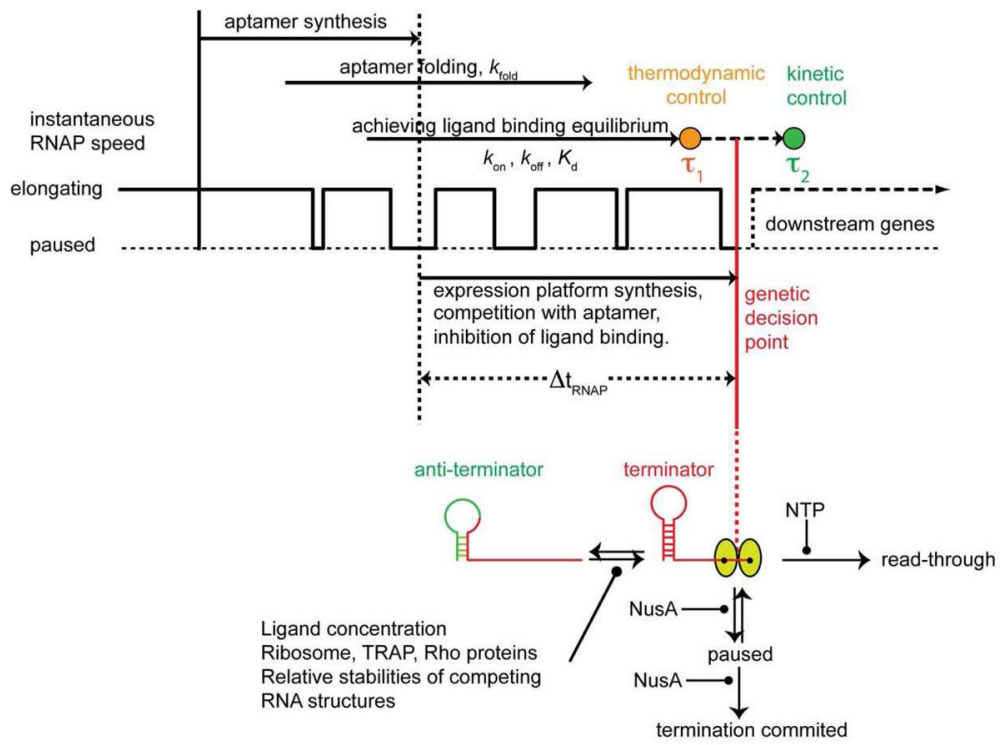

FIGURE 5.

Kinetic $v s$. thermodynamic control of riboswitches and ribozymes. Transcriptionally controlled riboswitches can operate under kinetic control (green dot) or thermodynamic control (orange dot). Upper panel depicts events relating to riboswitch control as horizontal lines with arrowheads (not to scale). $\Delta t_{\text {RNAP }}$ represents the time from the completion of aptamer synthesis to the genetic decision point (e.g. RNAP reaching a transcription terminator), shown as a vertical red line; $\tau$ represents the time constant for reaching ligand binding and dissociation equilibrium. When $\Delta \mathrm{t}_{\mathrm{RNAP}}<\tau\left(\tau_{2}\right.$, green), RNAP arrives at the terminator prior to ligand and aptamer reaching binding equilibrium, the riboswitch operates under kinetic control. When $\Delta \mathrm{t}_{\mathrm{RNAP}}>\tau\left(\tau_{1}\right.$, orange $)$, RNAP arrives at the terminator after ligand and aptamer reach equilibrium and the riboswitch exhibits thermodynamic control. Lower panel depicts a simplified kinetic scheme for competing events occurring at the terminator, depicted as a strong RNA hairpin followed by a slippery U-track. Two yellow ovals depict the RNAP active site. At the terminator, RNAPs are kinetically partitioned into species that read through the terminator or that enter a reversible pause before committing to termination. Nucleotide triphosphates (NTPs) and NusA modulate rates of these events. Additionally, the relative populations of terminator and antiterminator RNAs can be modulated by ligand concentration, ribosomes etc. 


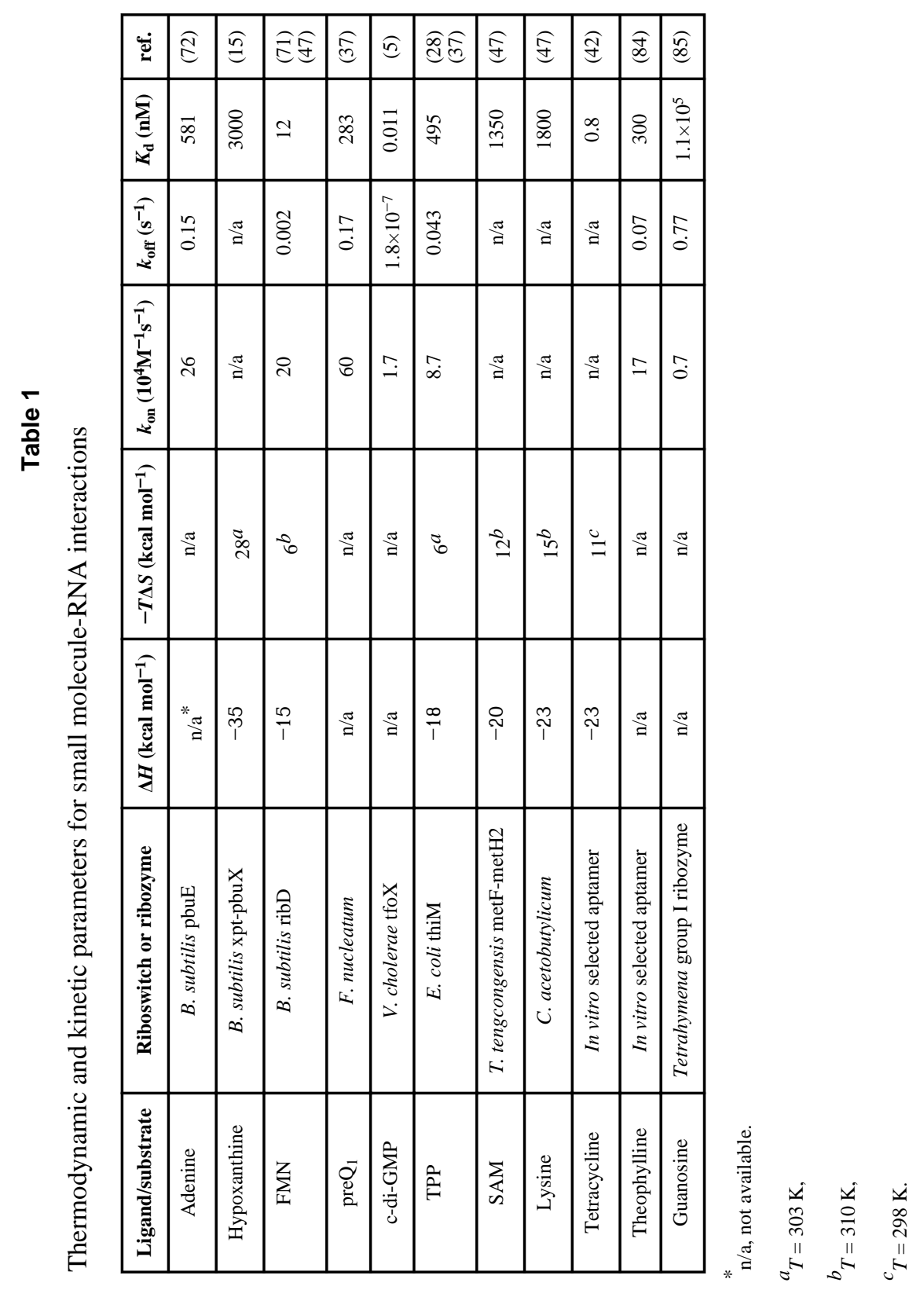

